\title{
EDIFICATE
}

I Congreso de Escuelas de Edificación y Arquitectura Técnica de España València, 4 y 5 de noviembre de 2021

Escuela Técnica Superior de Ingeniería de Edificación

Universitat Politècnica de València

Doi: https://doi.org/10.4995/EDIFICATE2021.2021.13295

\section{Convergencia de Títulos de Grado conforme a la Orden ECI $3855 / 2015$}

\section{Convergence of Bachelor Degrees according to Order ECI $3855 / 2015$}

\author{
Juan Manuel Santiago Zaragozaa ${ }^{a}$ María Segarra Cañamares ${ }^{b}$, \\ (en representación de todas las Escuelas miembros de la CODATIE que han \\ participado en la elaboración de los reconocimientos) \\ aPresidente CODATIE, Escuela Técnica Superior de Ingeniería de Edificación, Universidad de \\ Granada, santi@ugr.es b Secretaria CODATIE. Escuela Politécnica de Cuenca, Universidad de \\ Castilla-La Mancha, maría.segarra@uclm.es
}

\begin{abstract}
Current legislation establishes the profession of Technical Architect as a regulated profession that requires an official Bachelor's Degree obtained in accordance with article 12.9 of Royal Decree 1393/2007, in accordance with the conditions established in the Agreement of the Council of Ministers of 14 December 2007.
\end{abstract}

This Agreement entrusts the Minister of Education and Science with the establishment of the requirements regarding the planning of the courses. ORDER ECI/3855/2007, of 27 December 2007, establishes the requirements for the verification of the official university degrees that enable the exercise of the profession of Technical Architect.

The ORDEN ECl establishes that the syllabuses will have a duration of 240 European credits containing at least a basic training module of 60 credits, a specific training module of 108 credits and a final degree project of 12 credits. The Conference of Directors of University Centres that offer degrees in the field of technical architecture and building has worked to reach a consensus on the subjects subject matter subject to the recognition of the 108 credits of specific training that allow for the Convergence of the Degree Degrees of the different Universities.

Keywords: Study plan, validations, regulated profession, Technical Architecture, Universities. 


\begin{abstract}
Resumen
La legislación vigente conforma la profesión de Arquitecto Técnico como profesión regulada que requiere de un título oficial de Grado obtenido de acuerdo con el artículo 12.9 del Real Decreto 1393/2007, conforme a las condiciones establecidas en el Acuerdo de Consejo de ministros de 14 de diciembre de 2007.

Dicho Acuerdo encomienda al ministro de Educación y Ciencia el establecimiento de los requisitos respecto a la planificación de las enseñanzas. Siendo la ORDEN ECI/3855/2007, de 27 de diciembre, la que establece los requisitos para la verificación de los títulos universitarios oficiales que habiliten para el ejercicio de la profesión de Arquitecto Técnico.

La ORDEN ECl establece que los planes de estudios tendrán una duración de 240 créditos europeos conteniendo como mínimo un módulo de formación básica de 60 créditos, uno de formación específica de 108 créditos y uno de proyecto fin de grado de 12 créditos. Desde la Conferencia de directores y directoras de centros universitarios que imparten titulaciones del ámbito de la arquitectura técnica y la edificación, se ha trabajado para consensuar las asignaturas sujetas al reconocimiento de los 108 créditos de formación específica que permiten la Convergencia de los Títulos de Grado de las distintas Universidades.
\end{abstract}

Palabras clave: Plan de estudios, convalidaciones, profesión regulada, Arquitectura Técnica, Universidades. 


\section{Introducción}

La Orden ECI/3855/2007, de 27 de diciembre, por la que se establecen los requisitos para la verificación de los títulos universitarios oficiales que habiliten para el ejercicio de la profesión de Arquitecto Técnico, establece como Objetivos en su Apartado 3, las competencias que el estudiante debe adquirir.

Dichas competencias se enumeran en 8 apartados los cuales contemplan:

1. Dirigir la ejecución material de las obras de edificación, de sus instalaciones y elementos, llevando a cabo el control cualitativo y cuantitativo de lo construido mediante el establecimiento y gestión de los planes de control de materiales, sistemas y ejecución de obra, elaborando los correspondientes registros para su incorporación al Libro del Edificio. Llevar el control económico de la obra elaborando las certificaciones y la liquidación de la obra ejecutada.

2. Redactar estudios y planes de seguridad y salud laboral y coordinar la actividad de las empresas en materia de seguridad y salud laboral en obras de construcción, tanto en fase de proyecto como de ejecución.

3. Llevar a cabo actividades técnicas de cálculo, mediciones, valoraciones, tasaciones y estudios de viabilidad económica; realizar peritaciones, inspecciones, análisis de patología y otros análogos y redactar los informes, dictámenes y documentos técnicos correspondientes; efectuar levantamientos de planos en solares y edificios.

4. Elaborar los proyectos técnicos y desempeñar la dirección de obras de edificación en el ámbito de su habilitación legal.

5. Gestionar las nuevas tecnologías edificatorias y participar en los procesos de gestión de la calidad en la edificación; realizar análisis, evaluaciones y certificaciones de eficiencia energética, así como estudios de sostenibilidad en los edificios.

6. Dirigir y gestionar el uso, conservación y mantenimiento de los edificios, redactando los documentos técnicos necesarios. Elaborar estudios del ciclo de vida útil de los materiales, sistemas constructivos y edificios. Gestionar el tratamiento de los residuos de demolición y de la construcción.

7. Asesorar técnicamente en los procesos de fabricación de materiales y elementos utilizados en la construcción de edificios.

8. Gestionar el proceso inmobiliario en su conjunto. Ostentar la representación técnica de las empresas constructoras en las obras de edificación.

Todas estas competencias deben de ser adquiridas conforme a los tres módulos que marca la Orden ECl/3855/2007 en su Apartado 5 sobre Planificación de las enseñanzas Introducción.

60 créditos de formación básica, 108 créditos de formación específica y 12 créditos de Proyecto Fin de Grado. 


\section{Objetivo}

Dar a conocer, a todas las Escuelas que imparten titulaciones que habilitan para el ejercicio de la profesión de Arquitecto Técnico, el trabajo llevado a cabo desde la Conferencia de directores y directoras de centros universitarios que imparten titulaciones del ámbito de la arquitectura técnica y la edificación (CODATIE) en relación a la Convergencia de los títulos de Grado conforme a la Orden ECI 3855/2007 de 27 de diciembre.

\section{Desarrollo}

En base al acuerdo alcanzado por los jefes de estudios, en su reunión mantenida el 17 de junio de 2016, en relación a la necesidad de buscar la Convergencia de los títulos de Grado de las distintas universidades tanto en créditos, como en módulos, materias y asignaturas que quedan incluidos en la Orden ECI 3855/2007 de 27 de diciembre, la CODATIE establece la necesidad de consensuar las asignaturas que estarían sujetas a los 108 créditos y se hace petición a todas las Escuelas de su estudio.

Con este objetivo y tras haber recopilado toda la información recibida por las Escuelas, se muestran las propuestas en la reunión mantenida por la CODATIE el 13 de diciembre de 2017, lo que motiva que se convoque a los jefes de estudios de las Escuelas con objeto de definir los criterios básicos sobre los que trabajar.

Con fecha 2 de marzo de 2018 se mantuvo la reunión de jefes de estudios en la Escuela Técnica Superior de Ingeniería de Edificación de la Universitat Politècnica de València, a la que asistieron representantes de 13 escuelas.

En el programa de la reunión se tomó como punto del orden del día para trabajar:

- ORDEN ECI 3855/2007 de 27 de diciembre: Estudios de las asignaturas sujetas a convalidación. Análisis de las propuestas recibidas, resumen, conclusiones y medidas a adoptar.

En la reunión se deliberó sobre los créditos y asignaturas que debían reconocerse en cada módulo de formación específica y los márgenes que iban a aceptarse, teniendo en cuenta que las asignaturas tienen diferentes créditos en cada Escuela y hay diferencias entre los planes de estudio. También se revisaron las competencias, y las propuestas que había enviado cada escuela. Cada jefe de estudios expuso los ajustes que tendría que hacer para encajar la propuesta. Se acordó que además del reconocimiento de 108 créditos totales con los 6 bloques, podía ser posible el reconocimiento por bloques individuales entre escuelas.

\subsection{Acuerdos adoptados para el reconocimiento de créditos}

En relación con las asignaturas básicas del grado: se reconocerán todas las asignaturas Básicas que pertenezcan a la Rama de Ingeniería Arquitectura, con un máximo de 60 créditos. 
En cuanto a las asignaturas específicas: Si un/a alumno/a tiene completado un módulo en la universidad origen, de los seis que componen el plan de estudios, se intentará reconocer dicho módulo en la universidad destino. A continuación, se expone los requerimientos de máximos y mínimos sobre los créditos de cada uno de los módulos que se podrán reconocer en Bloque:

- Módulo de Expresión Gráfica: Máximo 12 ECTS y mínimo 9 ECTS

- Módulo de Técnicas y Tecnologías de la Edificación: Máximo 36 ECTS y mínimo 30 ECTS. (Se intentará que la parte de Materiales sea un $40 \%$ y la parte de Construcción sea un 60\%)

- Módulo de Estructuras e Instalaciones de la Edificación: Máximo 18 ECTS y mínimo 15 ECTS (Se intentará que la parte de Instalaciones sea un $66,67 \%$ y la parte de Estructuras sea un $33,33 \%$ )

- Módulo de Gestión del Proceso: Máximo 18 ECTS y mínimo 16,5 ECTS

- Módulo de Gestión Urbanística y Economías aplicadas: Máximo 18 ECTS y mínimo 14 ECTS

- Módulo de Proyectos Técnicos: Máximo 12 ECTS y mínimo 9 ECTS

La suma de los créditos de todos los módulos de formación específica tiene que ser, si se puede por tema de créditos, de 108 ECTS. El margen por módulo y sobre el total será de +3 créditos. En total, entre básicas y obligatorias, se intentará llegar a 168 ECTS.

\section{Resultados}

A fecha septiembre del 2019 son doce las universidades que ya han elaborado y aprobado por los órganos competentes las tablas de reconocimiento en base a las cuales se facilita la movilidad de los alumnos.

Dichas tablas cumplen con todos y cada uno de los acuerdos adoptados, tanto en el número de créditos por materia, como en la suma total de créditos, recogiendo en ellas las asignaturas con las cuales se adquieren las competencias establecidas en la Orden ECl.

A continuación, se muestran las tablas con la distribución de créditos por módulos y asignaturas de las doce Universidades. 
Convergencia de Títulos de Grado conforme a la Orden ECI 3855/2015-

Convergence of Bachelor Degrees according to Order ECI 3855/2015

\begin{tabular}{|c|c|c|c|c|}
\hline \multicolumn{5}{|c|}{ BÁSICOS } \\
\hline & Créditos ECTS minimos & \multicolumn{2}{|c|}{ Total Básicos } & 60,0 \\
\hline \multicolumn{5}{|c|}{ ESPECIFICOS } \\
\hline MODULO & Asignatura & Código & ECTS & Total/Módulo \\
\hline \multirow{2}{*}{ Expresión Gráfica } & Dibujo arquitectónico III & 6456 & 6,0 & \multirow{2}{*}{12,0} \\
\hline & Topografia y replanteos & 6452 & 6,0 & \\
\hline \multirow{6}{*}{$\begin{array}{c}\text { Téenicas y Tecnología } \\
\text { de la } \\
\text { Edificación }\end{array}$} & Materiales I & 6438 & 6,0 & \multirow{6}{*}{33,0} \\
\hline & Materiales II & 6442 & 6,0 & \\
\hline & Construcción I & 6438 & 6,0 & \\
\hline & Construcción II & 6443 & 6,0 & \\
\hline & Construcción III & 6448 & 6,0 & \\
\hline & Eficiencia Energética & 6474 & 3,0 & \\
\hline \multirow{3}{*}{$\begin{array}{c}\text { Estructuras e } \\
\text { Instalaciones de la } \\
\text { Edificación }\end{array}$} & Instalaciones II & 6453 & 6,0 & \multirow{3}{*}{15,0} \\
\hline & Instalaciones III & 6462 & 3,0 & \\
\hline & $\begin{array}{l}\text { Análisis de estructuras } y \text { fundamentos de hormigón } \\
\text { armado }\end{array}$ & 6458 & 6,0 & \\
\hline \multirow{4}{*}{ Gestión del Proceso } & Organización de obra & 6466 & 6,0 & \multirow{4}{*}{18,0} \\
\hline & Prevención y seguridad & 6460 & 6,0 & \\
\hline & Técnicas en prevención de riesgos laborales & 6467 & 3,0 & \\
\hline & Equipos de Obra y Medios Auxiliares & 6461 & 3,0 & \\
\hline \multirow{3}{*}{$\begin{array}{l}\text { Gestión Urbanística y } \\
\text { Economia aplicadas }\end{array}$} & Presupuestos I & 6459 & 6,0 & \multirow{3}{*}{18,0} \\
\hline & Gestión Económica y Urbanística & 6471 & 6,0 & \\
\hline & Peritaciones y Tasaciones & 6470 & 6,0 & \\
\hline \multirow{2}{*}{ Proyectos Técnicos } & Proyectos técnicos I & 6464 & 6,0 & \multirow{2}{*}{12,0} \\
\hline & Proyectos técnicos II & 6468 & 6,0 & \\
\hline & & \multicolumn{2}{|c|}{ Total Específicas } & 108,0 \\
\hline & & \multicolumn{2}{|c|}{ TOTAL RECONOCIBLES } & 168,0 \\
\hline
\end{tabular}

Fig. 1 Universidad de Burgos 


\begin{tabular}{|c|c|c|c|c|}
\hline \multicolumn{5}{|c|}{ BÁSICOS } \\
\hline & Créditos ECTS mínimos & \multicolumn{2}{|c|}{ Total Básicos } & 60,0 \\
\hline \multicolumn{5}{|c|}{ ESPECIFICOS } \\
\hline MODULO & Asignatura & Código & ECTS & Total/Módulo \\
\hline Expresión Gráfica & $\begin{array}{c}\text { Dibujo II } \\
\text { Topografia y replanteos }\end{array}$ & $\begin{array}{l}59312 \\
59323\end{array}$ & $\begin{array}{l}6,0 \\
6,0\end{array}$ & 12,0 \\
\hline \multirow{5}{*}{$\begin{array}{c}\text { Técnicas y Tecnología } \\
\text { de la } \\
\text { Edificación }\end{array}$} & Materiales de Construcción I & 59306 & 6,0 & \multirow{5}{*}{33,0} \\
\hline & Construcción I & 59307 & 6,0 & \\
\hline & Construcción III & 59316 & 9,0 & \\
\hline & Construcción IV & 59324 & 6,0 & \\
\hline & Patología y Restauración & 59325 & 6,0 & \\
\hline \multirow{2}{*}{$\begin{array}{c}\text { Estructuras e } \\
\text { Instalaciones de la }\end{array}$} & Instalaciones de la Edificación | & 59313 & 9,0 & \multirow{2}{*}{18,0} \\
\hline & Estructuras de la Edificación I & 59314 & 9,0 & \\
\hline \multirow{3}{*}{ Gestión del Proceso } & Prevención y Seguridad en el Trabajo & 59319 & 6,0 & \multirow{3}{*}{18,0} \\
\hline & Planificación, Organización y Control de Obras & 59326 & 6,0 & \\
\hline & Calidad en la Edificación & 59327 & 6,0 & \\
\hline \multirow{3}{*}{$\begin{array}{l}\text { Gestión Urbanistica y } \\
\text { Economia aplicadas }\end{array}$} & Mediciones y Presupuestos & 59329 & 6,0 & \multirow{3}{*}{18,0} \\
\hline & $\begin{array}{l}\text { Peritaciones } \mathrm{y} \text { Tasaciones } \\
\end{array}$ & 59331 & 6,0 & \\
\hline & Gestión Urbanistica y Construcciones Urbanas & 59328 & 6,0 & \\
\hline \multirow{3}{*}{ Proyectos Técnicos } & Introducción a la Prevención y Seguridad y & & & \multirow{3}{*}{12,0} \\
\hline & Proyectos Técnicos & $\frac{59317}{50318}$ & $\frac{6,0}{60}$ & \\
\hline & Proyectos Técnicos & & 6,0 & \\
\hline & & \multicolumn{2}{|c|}{ Total Especificas } & 111,0 \\
\hline & & \multicolumn{2}{|c|}{ TOTAL RECONOCIBLES } & 171,0 \\
\hline
\end{tabular}

Fi g. 2 Universidad de Castilla-La Mancha

\begin{tabular}{|c|c|c|c|c|}
\hline \multicolumn{5}{|c|}{ BÁSICOS } \\
\hline & Créditos ECTS mínimos & \multicolumn{2}{|c|}{ Total Básicos } & 60,0 \\
\hline \multicolumn{5}{|c|}{ ESPECIFICOS } \\
\hline MODULO & Asignatura & Código & ECTS & Total/Módulo \\
\hline \multirow{2}{*}{ Expresión Gráfica } & Expresión Gráfica Arquitectónica I & 670601008 & 6,0 & \multirow{2}{*}{12,0} \\
\hline & Geometria Descriptiva & 670601004 & 6,0 & \\
\hline \multirow{5}{*}{$\begin{array}{c}\text { Téenicas y Tecnología } \\
\text { de la } \\
\text { Edificación }\end{array}$} & Materiales I & $670 \mathrm{G} 01003$ & 6,0 & \multirow{5}{*}{30,0} \\
\hline & Materiales II & $670 \mathrm{G} 01012$ & 6,0 & \\
\hline & Construcción I & 670601009 & 6,0 & \\
\hline & Construcción II & $670 G 01011$ & 6,0 & \\
\hline & Construcción IIII & $670 G 01017$ & 6,0 & \\
\hline \multirow{3}{*}{$\begin{array}{c}\text { Estructuras e } \\
\text { Instalaciones de la } \\
\text { Edificación }\end{array}$} & Instalaciones II & $670 G 01024$ & 6,0 & \multirow{3}{*}{18,0} \\
\hline & Instalaciones III & $670 G 01035$ & 6,0 & \\
\hline & Estructuras I & $670 G 01019$ & 6,0 & \\
\hline \multirow{3}{*}{ Gestión del Proceso } & Seguridad y Prevención & $670 G 01031$ & 6,0 & \multirow{3}{*}{18,0} \\
\hline & Gestión de Calidad, Seguridad y Medio Ambiente & 670601032 & 6,0 & \\
\hline & Organización, Programación y Control & $670 G 01021$ & 6,0 & \\
\hline \multirow{3}{*}{$\begin{array}{l}\text { Gestión Urbanistica y } \\
\text { Economia aplicadas }\end{array}$} & Mediciones, Presupuestos y Control económico & 670601030 & 6,0 & \multirow{3}{*}{18,0} \\
\hline & Gestión inmobiliaria, tasaciones y peritaciones & 670601033 & 6,0 & \\
\hline & Derecho público de la edificación & $670 \mathrm{G} 01015$ & 6,0 & \\
\hline \multirow{2}{*}{ Proyectos Técnicos } & Proyectos téenicos I & $670 G 01023$ & 6,0 & \multirow{2}{*}{12,0} \\
\hline & Proyectos técnicos II & 670601027 & 6,0 & \\
\hline & & \multicolumn{2}{|c|}{ Total Especificas } & 108,0 \\
\hline & & \multicolumn{2}{|c|}{ TOTAL RECONOCIBLES } & 168,0 \\
\hline
\end{tabular}

Fig. 3 Universidad de la Coruña 
Convergencia de Títulos de Grado conforme a la Orden ECI 3855/2015 -

Convergence of Bachelor Degrees according to Order ECI 3855/2015

\begin{tabular}{|c|c|c|c|c|}
\hline \multicolumn{5}{|c|}{ BÁSICOS } \\
\hline & Créditos ECTS mínimos & \multicolumn{2}{|c|}{ Total Básicos } & 60,0 \\
\hline \multicolumn{5}{|c|}{ ESPECIFICOS } \\
\hline MODULO & Asignatura & Código & ECTS & Total/Módulo \\
\hline \multirow{2}{*}{ Expresión Gráfica } & Dibujo \|I & 502309 & 6,0 & \multirow[b]{2}{*}{12,0} \\
\hline & Topografia & 500986 & 6,0 & \\
\hline \multirow{5}{*}{$\begin{array}{c}\text { Técnicas y Tecnología } \\
\text { de la } \\
\text { Edificación }\end{array}$} & Materiales I & 500985 & 6,0 & \multirow{5}{*}{30,0} \\
\hline & Materiales II & 500990 & 6,0 & \\
\hline & Construcción I & 500983 & 6,0 & \\
\hline & Construcción II & 500987 & 6,0 & \\
\hline & Construcción III y control de calidad I & 500991 & 6,0 & \\
\hline \multirow{3}{*}{$\begin{array}{c}\text { Estructuras e } \\
\text { Instalaciones de la } \\
\text { Edificación }\end{array}$} & Instalaciones I & 500984 & 6,0 & \multirow{3}{*}{18,0} \\
\hline & Instalaciones II & 500989 & 6,0 & \\
\hline & Estructuras 1 & 502306 & 6,0 & \\
\hline \multirow{3}{*}{ Gestión del Proceso } & Organización y equipos de obra & 500993 & 6,0 & \multirow{3}{*}{18,0} \\
\hline & Legislación y Prevención I & 500992 & 6,0 & \\
\hline & Mantenimimiento || y control de calidad & 501008 & 6,0 & \\
\hline \multirow{3}{*}{$\begin{array}{l}\text { Gestión Urbanistica y } \\
\text { Economía aplicadas }\end{array}$} & $\begin{array}{l}\text { Presupuestos I } \\
\end{array}$ & 500997 & 6,0 & \multirow{3}{*}{18,0} \\
\hline & Presupuestos II & 501005 & 6,0 & \\
\hline & Peritaciones y Gestión Urbanistica & 500996 & 6,0 & \\
\hline \multirow{2}{*}{ Proyectos Técnicos } & Proyectos & 501007 & 6,0 & \multirow{2}{*}{12,0} \\
\hline & Ofiçina técnica & 500995 & 6,0 & \\
\hline & & \multicolumn{2}{|c|}{ Total Especificas } & 108,0 \\
\hline & & \multicolumn{2}{|c|}{ TOTAL RECONOCIBLES } & 168,0 \\
\hline
\end{tabular}

Fig. 4 Universidad de Extremadura

\begin{tabular}{|c|c|c|c|c|}
\hline \multicolumn{5}{|c|}{ BÁSICOS } \\
\hline & Créditos ECTS minimos & \multicolumn{2}{|c|}{ Total Básicos } & 60,0 \\
\hline \multicolumn{5}{|c|}{ ESPECIFICOS } \\
\hline MODULO & Asignatura & Código & ECTS & Total/Módulo \\
\hline Expresión Gráfica & $\begin{array}{l}\text { Expresión gráfica de la Tecnologia de la } \\
\text { Edificación } \\
\end{array}$ & 25 & 6,0 & \multirow[t]{2}{*}{12,0} \\
\hline \multirow{5}{*}{$\begin{array}{c}\text { Técnicas y Tecnología } \\
\text { de la } \\
\text { Edificación }\end{array}$} & $\begin{array}{c}\text { Topografia } \\
\text { Materiales II: Tecnologia de los Materiales }\end{array}$ & $3 \mathrm{~A}$ & 6,0 & \\
\hline & $\frac{\text { Materiales II: Tecnologia de los Materiales }}{\text { Materiales III: Ensayos y Control }}$ & $\frac{22}{26}$ & 6,0 & \multirow{4}{*}{30,0} \\
\hline & Construcción l: Historia, Tipologias y fundamentos de & 15 & 6,0 & \\
\hline & Construcción II: Sistemas Estructurales & 23 & 6,0 & \\
\hline & Construcción III: Sistemas Complementarios & 27 & 6,0 & \\
\hline \multirow{3}{*}{$\begin{array}{c}\text { Estructuras e } \\
\text { Instalaciones de la } \\
\text { Edificación }\end{array}$} & Estructuras I & 24 & 6,0 & \multirow{3}{*}{18,0} \\
\hline & Instalaciones I & 28 & 6,0 & \\
\hline & Instalaciones II & 34 & 6,0 & \\
\hline \multirow{3}{*}{ Gestión del Proceso } & Organización y Programación en Edificación & 32 & 6,0 & \multirow{3}{*}{18,0} \\
\hline & Prevención y Seguridad & 43 & 6,0 & \\
\hline & Gestión de la Calidad & 42 & 6,0 & \\
\hline \multirow{3}{*}{$\begin{array}{l}\text { Gestión Urbanistica y } \\
\text { Economia aplicadas }\end{array}$} & Proyecto de Desarrollo Urbano & 37 & 6,0 & \multirow{3}{*}{18,0} \\
\hline & Mediciones y Presupuestos & 36 & 6,0 & \\
\hline & Valoraciones, Tasaciones y Peritaciones & 45 & 6,0 & \\
\hline \multirow{3}{*}{ Proyectos Técnicos } & Proyecto de Gestión del Proceso y Equipos de & & & \multirow{3}{*}{12,0} \\
\hline & Obras & 44 & 6,0 & \\
\hline & Proyectos I: Ingeniería de Edificación Elemental & 31 & 6,0 & \\
\hline & & \multicolumn{2}{|c|}{ Total Especificas } & 108,0 \\
\hline & & \multicolumn{2}{|c|}{ IOTAL RECONOCIBLES } & 168,0 \\
\hline
\end{tabular}

Fig. 5 Universidad de Granada 


\begin{tabular}{|c|c|c|c|c|}
\hline \multicolumn{5}{|c|}{ BÁSICOS } \\
\hline & Créditos ECTS mínimos & \multicolumn{2}{|c|}{ Total Básicos } & 60,0 \\
\hline \multicolumn{5}{|c|}{ ESPECIFICOS } \\
\hline MODULO & Asignatura & Código & ECTS & Total/Módulo \\
\hline \multirow{2}{*}{ Expresión Gráfica } & Replantejaments i Topografia & 20367 & 6,0 & \multirow[b]{2}{*}{12,0} \\
\hline & Dibuix Assistit per Ordinador & 20360 & 6,0 & \\
\hline \multirow{6}{*}{$\begin{array}{c}\text { Técnicas y Tecnología } \\
\text { de la } \\
\text { Edificación }\end{array}$} & Construcció I. Història de la Construcció & 20354 & 6,0 & \multirow{6}{*}{36,0} \\
\hline & Materials II & 20363 & 6,0 & \\
\hline & Construcció II & 20364 & 6,0 & \\
\hline & Materials III & 20368 & 6,0 & \\
\hline & Construcció III & 20365 & 6,0 & \\
\hline & Construcció IV & 20377 & 6,0 & \\
\hline \multirow{3}{*}{$\begin{array}{c}\text { Estructuras e } \\
\text { Instalaciones de la } \\
\text { Edificación } \\
\end{array}$} & Estructures I & 20361 & 6,0 & \multirow{3}{*}{18,0} \\
\hline & Estructures II & 20366 & 6,0 & \\
\hline & |nstal-lacions | & 20362 & 6,0 & \\
\hline \multirow{3}{*}{ Gestión del Proceso } & Organització i Equips d'Obra & 20370 & 6,0 & \multirow{3}{*}{21,0} \\
\hline & Prevenció i Seguretat & 20372 & 9,0 & \\
\hline & Control de Qualitat & 20379 & 6,0 & \\
\hline \multirow{2}{*}{$\begin{array}{l}\text { Gestión Urbanistica y } \\
\text { Economía aplicadas }\end{array}$} & Amidaments, Pressuposts i Valoracions I & 20371 & 6,0 & \multirow{2}{*}{12,0} \\
\hline & Amidaments, Pressuposts i Valoracions II & 20375 & 6,0 & \\
\hline Proyectos Técnicos & Projectes Tècnics & 20380 & 9,0 & 9,0 \\
\hline & & \multicolumn{2}{|c|}{ Total Especificas } & 108,0 \\
\hline & & \multicolumn{2}{|c|}{ TOTAL RECONOCIBIES } & 168,0 \\
\hline
\end{tabular}

Fig. 6 Universidad de las Islas Baleares

\begin{tabular}{|c|c|c|c|c|}
\hline \multicolumn{5}{|c|}{ BÁSICOS } \\
\hline & Créditos ECTS minimos & \multicolumn{2}{|c|}{ Total Básicos } & 60,0 \\
\hline \multicolumn{5}{|c|}{ ESPECIFICOS } \\
\hline MODULO & Asignatura & Código & ECTS & Total/Módulo \\
\hline \multirow{2}{*}{ Expresión Gráfica } & Expresión Gráfica II & 26537 & 6,0 & \multirow{2}{*}{12,0} \\
\hline & Replanteos y Topografia & 26542 & 6,0 & \\
\hline \multirow{4}{*}{$\begin{array}{c}\text { Técnicas y Tecnología } \\
\text { de la } \\
\text { Edificación }\end{array}$} & Historia de la Construcción & 26547 & 6,0 & \multirow{4}{*}{31,5} \\
\hline & Construcción I & 26540 & 7,5 & \\
\hline & Construcción II & 26543 & 9,0 & \\
\hline & Construcción III & 26544 & 9,0 & \\
\hline \multirow{3}{*}{$\begin{array}{c}\text { Estructuras e } \\
\text { Instalaciones de la } \\
\text { Edificación }\end{array}$} & Estructuras I & 26560 & 6,0 & \multirow{3}{*}{18,0} \\
\hline & Instalaciones II & 26559 & 6,0 & \\
\hline & Estructuras II & 26561 & 6,0 & \\
\hline \multirow{3}{*}{ Gestión del Proceso } & Calidad de la Edificación & 26552 & 6,0 & \multirow{3}{*}{18,0} \\
\hline & Planificación, Organización y Control de obras & 26553 & 6,0 & \\
\hline & $\begin{array}{l}\text { Thiroducción a la Prevención, Seguridad y Salud y } \\
\text { Proyectos Técnicos }\end{array}$ & 26555 & 6,0 & \\
\hline \multirow{2}{*}{$\begin{array}{l}\text { Gestión Urbanistica y } \\
\text { Economia aplicadas }\end{array}$} & Gestión Urbanistica & 26538 & 6,0 & \multirow{2}{*}{15,0} \\
\hline & Mediciones y Presupuestos & 26549 & 9,0 & \\
\hline \multirow{2}{*}{ Proyectos Técnicos } & Proyectos Técnicos & 26550 & 6,0 & \multirow{2}{*}{12,0} \\
\hline & Expresión Gráfica III & 26541 & 6,0 & \\
\hline & & \multicolumn{2}{|c|}{ Total Especificas } & 106,5 \\
\hline & & \multicolumn{2}{|c|}{ TOTAL RECONOCIBLES } & 166,5 \\
\hline
\end{tabular}

Fig. 7 Universidad del País Vasco 
Convergencia de Títulos de Grado conforme a la Orden ECI 3855/2015 -

Convergence of Bachelor Degrees according to Order ECI 3855/2015

\begin{tabular}{|c|c|c|c|c|}
\hline \multicolumn{5}{|c|}{ BÁSICOS } \\
\hline & Créditos ECTS mínimos & \multicolumn{2}{|c|}{ Total Básicos } & 60,0 \\
\hline \multicolumn{5}{|c|}{ ESPECIFICOS } \\
\hline MODULO & Asignatura & Código & ECTS & Total/Módulo \\
\hline \multirow{4}{*}{ Expresión Gráfica } & Técnicas avanzadas de expresión gráfica & 310731 & 3,0 & \multirow{4}{*}{10,0} \\
\hline & Levantamiento y replanteos en la edificación & 310719 & 4,5 & \\
\hline & Taller 3: Gestión I & 310718 & 1,0 & \\
\hline & Taller 4: Análisis del edificio & 310724 & 1,5 & \\
\hline \multirow{12}{*}{$\begin{array}{c}\text { Técnicas y Tecnología } \\
\text { de la } \\
\text { Edificación }\end{array}$} & Materiales pétreos & 310710 & 3,0 & \multirow{12}{*}{38,0} \\
\hline & Materiales no pétreos & 310716 & 6,0 & \\
\hline & Introducción a la construcción & 310704 & 4,5 & \\
\hline & Construcción de estructuras & 310714 & 4,5 & \\
\hline & Construcción bajo rasante & 310720 & 4,5 & \\
\hline & Construcción de envolventes y acabados & 310726 & 4,5 & \\
\hline & Taller 1: Aprender de la construcción tradicional & 310706 & 3,0 & \\
\hline & Taller 2: Modelizar conceptos (BIM) & 310712 & 1,0 & \\
\hline & Taller 3: Gestión I & 310718 & 1,0 & \\
\hline & Taller 4: Análisis del edificio & 310724 & 2,5 & \\
\hline & Taller 8: Proyectos & 310741 & 1,5 & \\
\hline & Taller 9: Modelo final & 310742 & 2,0 & \\
\hline \multirow{4}{*}{$\begin{array}{c}\text { Estructuras e } \\
\text { Instalaciones de la } \\
\text { Edificación }\end{array}$} & Instalaciones de fluidos & 310721 & 3,0 & \multirow{4}{*}{19,5} \\
\hline & Instalaciones electromecánicas & 310728 & 6,0 & \\
\hline & Introducción a las estructuras & 310709 & 6,0 & \\
\hline & Estructuras de acero y hormigón & 310715 & 4,5 & \\
\hline \multirow{6}{*}{ Gestión del Proceso } & Planificación y organización de obra & 310732 & 7,5 & \multirow{6}{*}{20,0} \\
\hline & Coordinación de seguridad y salud laboral & 310738 & 4,5 & \\
\hline & Prevención de riesgos laborales & 310722 & 4,5 & \\
\hline & Taller 4: Análisis del edificio & 310724 & 0,5 & \\
\hline & Taller 8: Proyectos & 310741 & 1,0 & \\
\hline & Taller 9: Modelo final & 310742 & 2,0 & \\
\hline \multirow{2}{*}{$\begin{array}{l}\text { Gestión Urbanistica y } \\
\text { Economía aplicadas }\end{array}$} & Presupuestos y control de costes & 310733 & 7,5 & \multirow{2}{*}{12,0} \\
\hline & Gestión urbanistica & 310729 & 4,5 & \\
\hline \multirow{2}{*}{ Proyectos Técnicos } & Taller 8: Proyectos & 310741 & 6,5 & \multirow{2}{*}{8,5} \\
\hline & Taller 9: Modelo final & 310742 & 2,0 & \\
\hline & & \multicolumn{2}{|c|}{ Total Especificas } & 108,0 \\
\hline & & \multicolumn{2}{|c|}{ TOTAL RECONOCIBLES } & 168,0 \\
\hline
\end{tabular}

Fig. 8 Universidad Politécnica de Cataluña 


\begin{tabular}{|c|c|c|c|c|}
\hline \multicolumn{5}{|c|}{ BÁSICOS } \\
\hline & Créditos ECTS minimos & \multicolumn{2}{|c|}{ Total Básicos } & 63,5 \\
\hline \multicolumn{5}{|c|}{ ESPECIFICOS } \\
\hline MODULO & Asignatura & Código & ECTS & Total/Módulo \\
\hline \multirow{2}{*}{ Expresión Gráfica } & Dibujo Arquitectónico II & 10010 & 4,5 & \multirow{2}{*}{ 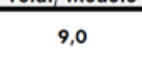 } \\
\hline & Topografia y Replanteos & 10011 & 4,5 & \\
\hline \multirow{6}{*}{$\begin{array}{c}\text { Técnicas y Tecnología } \\
\text { de la } \\
\text { Edificación }\end{array}$} & Materiales de Construcción II & 10012 & $\overline{4,5}$ & \multirow{6}{*}{36,0} \\
\hline & Materiales de Construcción IIII & 10013 & 9,0 & \\
\hline & Construcción I & 10014 & 9,0 & \\
\hline & Construcción II & 12484 & 4,5 & \\
\hline & Construcciones Históricas & 10019 & 4,5 & \\
\hline & Construcción IIII & 12485 & 4,5 & \\
\hline \multirow{3}{*}{$\begin{array}{c}\text { Estructuras e } \\
\text { Instalaciones de la } \\
\text { Edificación }\end{array}$} & Estructuras I & 10020 & 6,0 & \multirow{3}{*}{18,0} \\
\hline & Estructuras II & 10021 & 6,0 & \\
\hline & Instalaciones I| & 10022 & 6,0 & \\
\hline \multirow{4}{*}{ Gestión del Proceso } & Prevención y Seguridad I & 10024 & $\overline{4,5}$ & \multirow{4}{*}{21,0} \\
\hline & Prevención y Seguridad II & 10025 & 4,5 & \\
\hline & Organización, Programación y Control de Recursos & 10023 & 6,0 & \\
\hline & Calidad en la Edificación & 10026 & 6,0 & \\
\hline \multirow{3}{*}{$\begin{array}{l}\text { Gestión Urbanistica y } \\
\text { Economía aplicadas }\end{array}$} & Técnicas de Gestión Presupuestaria & 10030 & 6,0 & \multirow{3}{*}{15,0} \\
\hline & Peritaciones, Tasaciones y Valoraciones & 10029 & 4,5 & \\
\hline & Gestión Urbanistica & 10028 & 4,5 & \\
\hline \multirow{2}{*}{ Proyectos Técnicos } & Proyectos 1 & 10031 & 4,5 & \multirow{2}{*}{9,0} \\
\hline & Proyectos II & 10032 & 4,5 & \\
\hline & & \multicolumn{2}{|c|}{ Total Especificas } & 108,0 \\
\hline & & \multicolumn{2}{|c|}{ TOTAL RECONOCIBLES } & 171.5 \\
\hline
\end{tabular}

Fig. 9 Universitat Politècnica de València

\begin{tabular}{|c|c|c|c|c|}
\hline \multicolumn{5}{|c|}{ BÁSICOS } \\
\hline & Créditos ECTS mínimos & \multicolumn{2}{|c|}{ Total Básicos } & 60,0 \\
\hline \multicolumn{5}{|c|}{ ESPECIFICOS } \\
\hline MODULO & Asignatura & Código & ECTS & Total/Módulo \\
\hline \multirow{2}{*}{ Expresión Gráfica } & Topografia y replanteos & 28611 & 6,0 & \multirow{2}{*}{12,0} \\
\hline & Expresión gráfica de tecnologías constructivas & 28616 & 6,0 & \\
\hline \multirow{5}{*}{$\begin{array}{c}\text { Técnicas y Tecnología } \\
\text { de la } \\
\text { Edificación }\end{array}$} & Historia de la construcción & 28604 & 6,0 & \multirow{5}{*}{30,0} \\
\hline & Materiales I & 28607 & 6,0 & \\
\hline & Edificación I & 28609 & 6,0 & \\
\hline & Materiales II & 28613 & 6,0 & \\
\hline & Edificación II & 28614 & 6,0 & \\
\hline \multirow{3}{*}{$\begin{array}{c}\text { Estructuras e } \\
\text { Instalaciones de la } \\
\text { Edificación }\end{array}$} & Estructuras l: Introducción a las estructuras & 28612 & 6,0 & \multirow{3}{*}{18,0} \\
\hline & Instalaciones I & 28615 & 6,0 & \\
\hline & Instalaciones II & 28622 & 6,0 & \\
\hline \multirow{3}{*}{ Gestión del Proceso } & Organización, programación control obras & 28626 & 6,0 & \multirow{3}{*}{18,0} \\
\hline & Seguridad y salud laboral & 28627 & 6,0 & \\
\hline & Equipos de obra & 28620 & 6,0 & \\
\hline \multirow{3}{*}{$\begin{array}{l}\text { Gestión Urbanistica y } \\
\text { Economía aplicadas }\end{array}$} & Mediciones y presupuestos & 28624 & 6,0 & \multirow{3}{*}{18,0} \\
\hline & Gestión urbanistica e impacto ambiental & 28640 & 6,0 & \\
\hline & Valoraciones, peritaciones y tasaciones & 28630 & 6,0 & \\
\hline \multirow{2}{*}{ Proyectos Técnicos } & Proyectos técnicos I & 28633 & 6,0 & \multirow{2}{*}{12,0} \\
\hline & Proyectos técnicos II & 28634 & 6,0 & \\
\hline & & \multicolumn{2}{|c|}{ Total Especificas } & 108,0 \\
\hline & & \multicolumn{2}{|c|}{ TOTAL RECONOCIBLES } & 168,0 \\
\hline
\end{tabular}

Fig.10 Universidad de Zaragoza 
Convergencia de Títulos de Grado conforme a la Orden ECI 3855/2015 -

Convergence of Bachelor Degrees according to Order ECI 3855/2015

\begin{tabular}{|c|c|c|c|c|}
\hline \multicolumn{5}{|c|}{ BÁSICOS } \\
\hline & Créditos ECTS mínimos & \multicolumn{2}{|c|}{ Total Básicos } & 60,0 \\
\hline \multicolumn{5}{|c|}{ ESPECIFICOS } \\
\hline MODULO & Asignatura & Código & ECTS & Total/Módulo \\
\hline \multirow{3}{*}{ Expresión Gráfica } & Dibujo Arquitectónico II & 545000010 & 3,0 & \multirow{3}{*}{12,0} \\
\hline & Geometría Descriptiva II & 545000011 & 3,0 & \\
\hline & Topografia I & 545000019 & 6,0 & \\
\hline \multirow{8}{*}{$\begin{array}{c}\text { Técnicas y Tecnología } \\
\text { de la } \\
\text { Edificación }\end{array}$} & Materiales de Construcción I & 545000008 & 6,0 & \multirow{8}{*}{36,0} \\
\hline & Introducción a la Construcción & 545000009 & 6,0 & \\
\hline & Construcción de Estructuras de Acero & 545000022 & 3,0 & \\
\hline & Onstrucción de Cerramientos de Fachadas y Cubierta & 545000026 & 6,0 & \\
\hline & Construcción Sostenible & 545000036 & 3,0 & \\
\hline & Historia de la Construcción & 545000028 & 3,0 & \\
\hline & Patología y Rehabilitación & 545000039 & 6,0 & \\
\hline & Mantenimiento & 545000045 & 3,0 & \\
\hline \multirow{3}{*}{$\begin{array}{c}\text { Estructuras e } \\
\text { Instalaciones de la } \\
\text { Edificación }\end{array}$} & Resistencia de Materiales y Elasticidad & 545000020 & 6,0 & \multirow{3}{*}{18,0} \\
\hline & Análisis de Estructuras y Geotecnia & 545000024 & 6,0 & \\
\hline & Instalaciones $\mid$ & 545000021 & 6,0 & \\
\hline \multirow{4}{*}{ Gestión del Proceso } & Calidad de la Edificación & 545000044 & 6,0 & \multirow{4}{*}{21,0} \\
\hline & Planificación y Programación de Obras I & 545000033 & 6,0 & \\
\hline & Gestión del Proceso Edificatorio & 545000041 & 3,0 & \\
\hline & Prevención y Seguridad I & 545000034 & 6,0 & \\
\hline \multirow{3}{*}{$\begin{array}{l}\text { Gestión Urbanística y } \\
\text { Economía aplicadas }\end{array}$} & Legislación Urbanística & 545000046 & 3,0 & \multirow{3}{*}{12,0} \\
\hline & Mediciones y Presupuestos & 545000032 & 6,0 & \\
\hline & Valoraciones y Tasaciones. Peritaciones Judiciales & 545000042 & 3,0 & \\
\hline \multirow{2}{*}{ Proyectos Técnicos } & Proyectos Técnicos I & 545000030 & 3,0 & \multirow{2}{*}{9,0} \\
\hline & Proyectos Técnicos II & 545000037 & 6,0 & \\
\hline & & \multicolumn{2}{|c|}{ Total Especificas } & 108,0 \\
\hline & & \multicolumn{2}{|c|}{ TOTAL RECONOCIBLES } & 168,0 \\
\hline
\end{tabular}

Fig.11 Universidad Politécnica de Madrid 


\begin{tabular}{|c|c|c|c|c|}
\hline \multicolumn{5}{|c|}{ BÁSICOS } \\
\hline & Créditos ECTS mínimos & \multicolumn{2}{|c|}{ Total Básicos } & 60,0 \\
\hline \multicolumn{5}{|c|}{ ESPECIFICOS } \\
\hline MODULO & Asignatura & Código & ECTS & Total/Módulo \\
\hline \multirow{2}{*}{ Expresión Gráfica } & Análisis e interpretación del proy. arquitectónico & 12660 & 4,5 & \multirow{2}{*}{9,0} \\
\hline & Topografia y replanteos & 12652 & 4,5 & \\
\hline \multirow{8}{*}{$\begin{array}{c}\text { Técnicas y Tecnología } \\
\text { de la } \\
\text { Edificación }\end{array}$} & Materiales de construcción I & 12645 & 4,5 & \multirow{8}{*}{34,5} \\
\hline & Materiales de construcción II & 12650 & 3,0 & \\
\hline & Introducción a la construcción & 12677 & 4,5 & \\
\hline & Construcción I & 12651 & 4,5 & \\
\hline & Construcción II & 12659 & 4,5 & \\
\hline & Construcción III & 12663 & 4,5 & \\
\hline & Historia de la construcción & 12688 & 4,5 & \\
\hline & Construcción industrializada. Construcción sostenible & 12683 & 4,5 & \\
\hline \multirow{4}{*}{$\begin{array}{c}\text { Estructuras e } \\
\text { Instalaciones de la } \\
\text { Edificación }\end{array}$} & Introducción a las estructuras de edificación & 12654 & 4,5 & \multirow{4}{*}{18,0} \\
\hline & Estructuras de edificación I & 12661 & 4,5 & \\
\hline & Estructuras de edificación II & 12662 & 4,5 & \\
\hline & Instalaciones de edificación I & 12665 & 4,5 & \\
\hline \multirow{4}{*}{ Gestión del Proceso } & Seguridad, salud y prevención de riesgos laborales & 12669 & 4,5 & \multirow{4}{*}{18,0} \\
\hline & Técnicas de organización y programación de la edif. & 12681 & 4,5 & \\
\hline & Gestión y aseguramiento de la calidad & 12672 & 4,5 & \\
\hline & Deontologia, organización y ejercicio profesional & 12684 & 4,5 & \\
\hline \multirow{4}{*}{$\begin{array}{l}\text { Gestión Urbanistica y } \\
\text { Economia aplicadas }\end{array}$} & Valoraciones y tasaciones & 12674 & 4,5 & \multirow{4}{*}{16,5} \\
\hline & Mediciones y presupuestos & 12680 & 4,5 & \\
\hline & Análisis y control de costes durante el proceso constr. & 12687 & 4,5 & \\
\hline & Gestión medioambiental y urbanística & 12682 & 3,0 & \\
\hline \multirow{2}{*}{ Proyectos Técnicos } & Proyectos técnicos I & 12679 & 4,5 & \multirow{2}{*}{9,0} \\
\hline & Proyectos técnicos II & 12686 & 4,5 & \\
\hline & & \multicolumn{2}{|c|}{ Total Especificas } & 105,0 \\
\hline & & \multicolumn{2}{|c|}{ TOTAL RECONOCIBLES } & 165,0 \\
\hline
\end{tabular}

Fig.12 Universidad Católica San Antonio de Murcia

\section{Conclusiones}

Tras tres años de trabajo, en las que las distintas escuelas que conforman la CODATIE han podido hacer una puesta en común sobre las equivalencias entre los distintos planes de estudios, se consiguió finalmente llegar a un acuerdo sobre los créditos que podrían ser reconocidos.

Este acuerdo facilita la movilidad entre escuelas y la posibilidad de conformar planes de estudios comunes, a la vez que diferentes en su ámbito de especialización, lo que supone un gran avance a la vez que una oportunidad.

Dar a conocer este trabajo a todas las Escuelas, y que estas se sumen al acuerdo adoptado, se considera una labor más de la CODATIE que se suma al trabajo ya realizado. 


\section{Referencias}

España. Real Decreto 1393/2007, de 29 de octubre, por el que se establece la ordenación de las enseñanzas universitarias oficiales. BOE, 30 de octubre de 2007, núm. 260, p. 44037 a 44048.

España. Resolución de 17 de diciembre de 2007, de la Secretaría de Estado de Universidades e Investigación, por la que se publica el Acuerdo de Consejo de Ministros de 14 de diciembre de 2007, por el que se establecen las condiciones a las que deberán adecuarse los planes de estudios conducentes a la obtención de títulos que habiliten para el ejercicio de la profesión regulada de Arquitecto Técnico. BOE, 21 de diciembre de 2007, núm. 305, p. 52848 a 52849.

España. Orden ECI/3855/2007, de 27 de diciembre, por la que se establecen los requisitos para la verificación de los títulos universitarios oficiales que habiliten para el ejercicio de la profesión de Arquitecto Técnico. BOE, 29 de diciembre de 2007, núm. 312, p. 53739 a 53742. 